\title{
Silicon alleviates salt and drought stress of Glycyrrhiza uralensis plants by improving photosynthesis and water status
}

\author{
W.J. ZHANG ${ }^{1,2}$, X.J. ZHANG ${ }^{1}$, D.Y. LANG ${ }^{3}$, M. $\mathrm{LI}^{4}$, H. LIU ${ }^{4}$, and X.H. ZHANG ${ }^{1,2 *}$ \\ College of Pharmacy, Ningxia Medical University, Yinchuan 750004, P.R. China ${ }^{1}$ \\ Laboratory of Hui Ethnic Medicine Modernization, Ningxia Medical University, Yinchuan 750004, P.R. China ${ }^{2}$ \\ Laboratory Animal Center, Ningxia Medical University, Yinchuan 750004, P.R. China ${ }^{3}$ \\ Desertification Control Institute, Ningxia Academy of Agriculture and Forestry Sciences, \\ Yinchuan 750002, P.R. China ${ }^{4}$
}

\begin{abstract}
Silicon has been widely reported to have a beneficial effect on improving plant tolerance to biotic and abiotic stresses. However, the mechanisms of Si in mediating responses to simultaneous salt and drought stresses are still poorly understood. Glycyrrhiza uralensis Fisch. is classified as a non-Si accumulator and suffered from salt and drought stresses. In this study, we investigated the long-term application of Si on Si content in G. uralensis roots, stems and leaves, leaf anatomy, ultrastructure, chlorophyll (Chl) content, gas exchange characteristics, relative water content, and growth of two-year-old plants under different salt and drought stresses. Silicon application resulted in a higher Si uptake in G. uralensis roots and more Si accumulation in leaves (especially deposition of Si on cell walls), and Si counteracted the adverse effects induced by salt and drought stresses on the leaf anatomy and ultrastructure. In plants treated with $\mathrm{Si}$, a higher chlorophyll content, net photosynthetic rate and relative water content led to a higher growth rate and dry mass under salt and drought stresses compared with corresponding non-Si treated plants.
\end{abstract}

Additional key words: chlorophyll, leaf ultrastructure, net photosynthetic rate, relative water content, stomatal conductance, transpiration rate, water use efficiency.

\section{Introduction}

Salt and drought stresses are two most critical abiotic threats that affect plant growth and production worldwide (Rizwan et al. 2015) and are becoming more serious concerns as world desertification increases (Janz et al. 2012). Furthermore, salinization and desertification are rapidly increasing on a global scale and currently affect more than $10 \%$ of arable land which results in more than $50 \%$ reduction in average yields of major crops ( $\mathrm{Li}$ et al. 2010, Ouzounidou et al. 2014). Generally, high salt concentration in the soil induces high osmotic potential of soil which results in water deficit within plants firstly, and drought stress usually occurred in the field together with salt stress would further enhance the moisture loss (Farooq et al. 2009).

Water deficit in plants usually reduces leaf expansion, impaired photosynthetic machinery, induces premature leaf senescence leading to reduction in dry matter accumulation (Bijanzadeh and Emam 2010, Aminian et al. 2011). In addition, plant tolerance to salt and drought stress is mainly associated with the maintenance of plant water status either by reducing water loss through decreasing transpiration or improving plant root capacity to extract more water through osmotic adjustment. Above all, salt and drought stresses mainly affect the photosynthetic characteristics and the water status of plants (Maghsoudi and Maghsoudi 2008, Shahid et al. 2014). They induce the decrease of net photosynthetic rate and chlorophyll (Chl) content, relative water content (RWC), and water use efficiency (WUE) (Peng et al. 2006, Jaleel et al. 2009, Ouzounidou et al. 2014, Abbas et al. 2015).

Glycyrrhiza uralensis Fisch. is a very popular herbal plant widely appreciated as Chinese medicine. Moreover,

Submitted 29 January 2019, last revision 10 October 2019, accepted 1 November 2019.

Abbreviations: $\mathrm{c}_{\mathrm{i}}$ - intercellular $\mathrm{CO}_{2}$ concentration; Chl - chlorophyll; DAT - days after treatment; E - transpiration rate; FWC - field water content; $g_{s}$ - stomatal conductance; $P_{N}$ - net photosynthetic rate; RWC - relative water content; WUE - water use efficiency.

Acknowledgements: The authors are grateful for the financial support provided by the project of the National Natural Science Foundation of China $(31460330,31860343)$ and the Key National Research and Development Programs (2017YFC1700706) and the Ningxia Natural Science Foundation (2019AAC03098). The first two authors contributed equally to this manuscript.

* Corresponding author; e-mail: zhang2013512@163.com. 
it is commonly used as additives in tobacco and food worldwide (Egamberdieva et al. 2016). It is also very important for wind hampering, sand fixing, and soil formation in arid and semiarid regions ( $\mathrm{Li}$ and Wang 2002). G. uralensis is often cultivated in arid, semiarid, and salt-affected regions. Therefore, improving the tolerance of commercial cultivars of $G$. uralensis to salt and drought stress has become a key target for sustainable development.

At present, there are various physical and chemical ways for improving the plant responses to abiotic stresses. Exogenous $\mathrm{Si}$ is one of the important methods. Its uptake by plants has been adopted as an effective strategy for alleviating the negative effects of salt and drought stress and improving salt and drought tolerance of plants (Zhu and Gong 2014). And Si may be a "quasi-essential" element for plants has been increasingly recognized (Pilon-Smits et al. 2009). However, its role in plant biology has not been understood clearly. In most cases, the favourable effects of Si seem to originate from reinforcement of the cell walls due to the deposition of $\mathrm{Si}$ in the form of amorphous silica and opal phytoliths (Epstein 1999). Meanwhile, its active involvement in a multitude of physiological and metabolic processes is also evident (Moussa 2006). Under drought stress, Si acts not only as a physical or mechanical barrier to minimize transpiration losses but also participates in many processes which improve drought tolerance (Hattori et al. 2005). Feng et al. (2010) found that Si protected the photosynthetic apparatus by protection of thylakoid membranes and improved pigment content, resulting in higher photosynthetic rate in cadmium stressed cucumber plants. Previous studies by Hattori et al. (2008) and Chen et al. (2011b) showed that the Si-induced alleviation of osmotic stress in cucumber and drought stress in rice is independent of leaf water status, but Wang et al. (2015) found that $\mathrm{Si}$ enhances the salt tolerance of cucumber by improving water balance. Above mentioned researchers studied the effects of Si on photosynthesis and water status of plants under every stress individually and in a short term, and they focused on grain crops, vegetables, and fruit trees. Nevertheless, salt and drought stresses usually occurred together and in a long term; the mechanisms of plant tolerance to salt and drought stress are physiologically connected and overlapped as mentioned above.

Our previous study addressed that $\mathrm{Si}$ can promote the growth of $G$. uralensis seedlings under salt and drought stresses by regulating antioxidant metabolism, osmoprotectants, ion balance, and so on ( $\mathrm{Li}$ et al. 2016, Zhang et al. 2017). These observations are consistent with many other non Si-accumulating plants (Katz 2014, Zhu and Gong 2014). In the present study, we studied the influence of Si on long term salt and drought stressed G. uralensis plants by analyzing the changes in Si content, leaf anatomical structure, Chl content, gas exchange parameters, water status, growth, and the dry matter accumulation. We hypothesized that $\mathrm{Si}$ application can increase Si content in roots and leaves, keep the integrity of leaves, increase $\mathrm{Chl}$ content, net photosynthetic rate and WUE, and thereby enhance growth and dry matter accumulation of $G$. uralensis under salt and drought stresses.

\section{Materials and methods}

Plants and experimental design: One-year-old Glycyrrhiza uralensis Fisch. plants were collected from Yanchi County, Ningxia Hui Autonomous Region, China. Pot experiment was conducted at Ningxia Medical University during growing season of 2016. Average day/ night temperatures were $28 / 16^{\circ} \mathrm{C}$, a 14 -h photoperiod, and natural irradiance. On April 18, 2016, G. uralensis plants were transplanted into pots containing sandy loam soil ( $\mathrm{pH} 7.4$, containing [ $\mathrm{g} \mathrm{kg}^{-1}$ ]: 3.28 organic matter, 0.87 total nitrogen, 2.5 total phosphorus, 0.17 instant potassium, 0.021 instant phosphorus, 0.053 alkaline solution nitrogen, 0.039 silica, and $2.3 \mathrm{NaCl}$ ).

Treatments were arranged in a randomized experimental design and included control (CK) containing $2.3 \mathrm{~g}(\mathrm{NaCl}) \mathrm{kg}^{-1}$ (dry soil), salt stress solely (S) containing $6 \mathrm{~g}(\mathrm{NaCl}) \mathrm{kg}^{-1}$ (dry soil $)+60-65 \%$ field water content (FWC), salt stress combined with drought stress (SD1) containing $6 \mathrm{~g}(\mathrm{NaCl}) \mathrm{kg}^{-1}$ (dry soil $)+45-50 \% \mathrm{FWC}$ or (SD2) $6 \mathrm{~g}(\mathrm{NaCl}) \mathrm{kg}^{-1}$ (dry soil) $+30-35 \%$ FWC with or without $0.1 \mathrm{~g}\left(\mathrm{SiO}_{2}\right) \mathrm{kg}^{-1}$ (dry soil). Silicon was applied as $\mathrm{K}_{2} \mathrm{SiO}_{3}$ and a corresponding amount of $\mathrm{K}$ (as $\mathrm{KCl}$ ) was also added to $\mathrm{CK}$ to compensate the $\mathrm{K}$ content. Drought was imposed by withholding water. The volume of water required to achieve the pot field capacity represented the consumption of water during the previous day. There were three replications per treatment and each replication comprised three pots, each pot included four plants. All pots were randomly arranged and periodically rotated to minimize the effects of environmental heterogeneity. At 70 and $110 \mathrm{~d}$ after drought treatment, G. uralensis plants were collected for evaluation of various physiological and biochemical parameters and growth parameters. Gas exchange parameters were measured at $90 \mathrm{~d}$ after treatment.

Determination of Si content: Si content of G. uralensis plants was determined colorimetrically by the molybdate method (Zhang et al. 2017). About $0.01 \mathrm{~g}$ of finely dry plant samples were placed in $100-\mathrm{cm}^{3}$ polyethylene tubes previously washed with $0.1 \mathrm{M} \mathrm{NaOH}$ and rinsed with demineralised water. $\mathrm{NaOH}\left(3 \mathrm{~cm}^{3}, 50 \%\right)$ was added to each tube and the suspensions autoclaved for $1 \mathrm{~h}$ to oxidize any organic matter present and soluble Si. To develop the colour, $35 \mathrm{~cm}^{3}$ of $200 \mathrm{~cm}^{3} \mathrm{dm}^{-3}$ acetic acid was added to $10 \mathrm{~cm}^{3}$ of ammonium molybdate solution, $5 \mathrm{~cm}^{3}$ of $200 \mathrm{~cm}^{3} \mathrm{dm}^{-3}$ tartaric acid, $1 \mathrm{~cm}^{3}$ of reducing solution and excess of $200 \mathrm{~cm}^{3} \mathrm{dm}^{-3}$ acetic acid to bring the volume to $50 \mathrm{~cm}^{3}$. After mixing, the absorbance was read at $630 \mathrm{~nm}$ with a spectrophotometer. Plastic ware rinsed with $0.1 \mathrm{M}$ $\mathrm{NaOH}$ for removing silica was used throughout the assay. Different amounts of silicic acid were included as standards for determining Si content of plant samples.

Ultrastructural observations of mesophyll cells: Three leaves per treatment were collected to examine chloroplast ultrastructure in mesophyll cells. A rectangular section of a leaf $(3.0 \times 1.5 \mathrm{~mm})$ near the center vein of each leaf was removed from a blade. After fixation with $2.5 \%$ 
$(\mathrm{m} / \mathrm{v})$ glutaraldehyde for $4 \mathrm{~h}$, and washed with $0.1 \mathrm{M}$ phosphate buffer, $\mathrm{pH}$ 7.4, leaf cells were post-fixed with $1 \%(\mathrm{~m} / \mathrm{v})$ osmic acid at $4{ }^{\circ} \mathrm{C}$ for $4 \mathrm{~h}$, washed with $0.1 \mathrm{M}$ phosphate buffer, and then dehydrated with acetone gradient (acetone:resin mixtures at 3:1, 1:1, and 1:3, v/v) and then placed in pure resin for $12 \mathrm{~h}$ at $37^{\circ} \mathrm{C}, 12 \mathrm{~h}$ at $45^{\circ} \mathrm{C}$, and $48 \mathrm{~h}$ at $60{ }^{\circ} \mathrm{C}$. The thin sections were cut from leaf samples with an $L K B-V$ ultramicrotome and placed upon 250 mesh grids. Samples were double stained using stem uranyl acetate and lead citrate and then observed and randomly photographed using a Hitachi-600 (Tokyo, Japan) transmission electron microscope (Xu et al. 2008).

Determination of $\mathrm{Chl}$ content: Chlorophyll was extracted from $0.3 \mathrm{~g}$ small pieces of leaf tissue in $20 \mathrm{~cm}^{3}$ acetone solution (acetone and ethanol, 2:1, v/v). After extraction at room temperature and under dark for $24 \mathrm{~h}, \mathrm{Chl}$ content in the supernatant was analyzed by measuring absorbance at 663 and $645 \mathrm{~nm}$, respectively, as described by Ming et al. (2007): Chl $a=12.72 \mathrm{~A}_{663}-2.59 \mathrm{~A}_{645}$; Chl $b=22.88 \mathrm{~A}_{645}-$ $4.67 \mathrm{~A}_{663} ; \mathrm{Chl} a+b=20.29 \mathrm{~A}_{645}+8.05 \mathrm{~A}_{663}$. All absorbances were measured with a $S P-752 P C$ spectrophotometer (Shanghai Spectrum Instruments, Shanghai, China).

Measurement of gas exchange characteristics: Net photosynthetic rate $\left(\mathrm{P}_{\mathrm{N}}\right)$, stomatal conductance $\left(\mathrm{g}_{\mathrm{s}}\right)$, transpiration rate $(\mathrm{E})$, and intercellular $\mathrm{CO}_{2}$ concentration $\left(\mathrm{c}_{\mathrm{i}}\right)$ of G. uralensis leaves were measured between 10:00 and 12:00 $\mathrm{h}$ on three consecutive days (20 - 22 August) using a portable photosynthesis system (CI-310, CID Bio-Science, Camas, WA, USA), using an infrared gas analyzer. Six similar, healthy, fully expanded leaves on the top of the stem in each treatment were measured at a leaf temperature of $20 \pm 2{ }^{\circ} \mathrm{C}$, an air humidity of $14 \pm 2 \%$, an irradiance of $570 \mu \mathrm{mol} \mathrm{m} \mathrm{m}^{-2} \mathrm{~s}^{-1}$, and $\mathrm{CO}_{2}$ concentration of $420 \mu \mathrm{mol} \mathrm{mol}^{-1}$. The WUE was calculated as the ratio of $\mathrm{P}_{\mathrm{N}}$ and $\mathrm{E}$.

Determination of water status: Fully expanded third leaf from the top of the main stem of three plants from each treatment were selected to measure the RWC. After measuring the fresh mass (FM), leaves were placed in distilled water for $24 \mathrm{~h}$ at $4{ }^{\circ} \mathrm{C}$ in darkness and the water saturated mass (WSM) was recorded. The samples were oven-dried at $60^{\circ} \mathrm{C}$ until constant mass was attained and leaf dry mass (DM) was determined. RWC was calculated based on the formula suggested by Teulat et al. (2003) as follows: $\mathrm{RWC}[\%]=[(\mathrm{FM}-\mathrm{DM}) /(\mathrm{WSM}-\mathrm{DM})] \times 100$.

Measurement of growth parameters: At the end of the experiment, the plant height, root height, root diameter, and stem diameter were recorded from three replicates of each treatment. The plant height was measured from the base of the stem, at the soil level, to the terminal bud of the main stem. The stem and root diameter were measured with a digital micrometer. Leaf area was obtained with a CI-203 leaf area scanner (CID Bio-Science, Camas, WA, USA). All plants were taken out of the soil and separated into leaves, stems, and roots. The tissues were first cleaned using distilled water. Then the sample was oven-dried at $60{ }^{\circ} \mathrm{C}$ for $48 \mathrm{~h}$ to constant mass and weighted.

Statistical analysis: All experimental data were analyzed by $A N O V A$ and Pearson's correlation analysis using SPSS 17.0 software (SPSS, Chicago, IL, USA). Significant differences were tested using the least significant difference (LSD) test at $P<0.05$. Mean values and standard errors (SEs) were presented $(n=6)$.

\section{Results}

At $70 \mathrm{~d}$ after treatment (DAT), Si content in G. uralensis roots significantly decreased under SD2 and Si content in stems significantly decreased under all treatments without $\mathrm{Si}$ addition compared to control (Table 1). At 110 DAT, $\mathrm{Si}$ content in roots and leaves significantly decreased under SD1 and SD2 compared with control when $\mathrm{Si}$ absence. However, Si addition significantly affected Si content in roots, stems, and leaves, and this effect was

Table 1. Effect of $\mathrm{Si}\left[0.1 \mathrm{~g}\left(\mathrm{SiO}_{2}\right) \mathrm{kg}^{-1}\right.$ (dry soil) $]$ on Si content in roots, stems, and leaves of Glycyrrhiza uralensis plants undercontrol conditions $(\mathrm{CK})$, salinity, and drought stress $\left[6 \mathrm{~g}(\mathrm{NaCl}) \mathrm{kg}^{-1}(\right.$ soil $)+60-65 \%$ field water content $(\mathrm{FWC})(\mathrm{S}), 6 \mathrm{~g}(\mathrm{NaCl}) \mathrm{kg}^{-1}(\mathrm{soil})$ $+45-50 \%$ FWC (SD1), $6 \mathrm{~g}(\mathrm{NaCl}) \mathrm{kg}^{-1}$ (soil) $+30-35 \%$ FWC (SD2)]. Means $\pm \mathrm{SEs}, n=6$; in the same column different capital letters indicate significant differences among different treatments without Si at 0.05 probability, and different lower case letters indicate significant differences among different treatments after Si application at 0.05 probability; $*$ stands for significant differences at 0.05 probability between two treatments with or without $\mathrm{Si}$.

\begin{tabular}{|c|c|c|c|c|c|c|}
\hline \multirow[t]{2}{*}{ Treatments } & \multicolumn{2}{|l|}{$\begin{array}{l}\text { Si content in roots } \\
{\left[\mathrm{mg} \mathrm{g}^{-1} \text { d.m. }\right]}\end{array}$} & \multicolumn{2}{|c|}{$\begin{array}{l}\text { Si content in stems } \\
{\left[\mathrm{mg} \mathrm{g}^{-1} \text { d.m. }\right]}\end{array}$} & \multicolumn{2}{|c|}{$\begin{array}{l}\text { Si content in leaves } \\
{\left[\mathrm{mg} \mathrm{g}^{-1} \text { d.m. }\right]}\end{array}$} \\
\hline & 70 DAT & 110 DAT & $70 \mathrm{DAT}$ & 110 DAT & $70 \mathrm{DAT}$ & 110 DAT \\
\hline $\mathrm{CK}$ & $4.23 \pm 0.30 \mathrm{~A}$ & $4.55 \pm 0.11 \mathrm{~A}$ & $3.50 \pm 0.15 \mathrm{~A}$ & $3.21 \pm 0.15 \mathrm{~A}^{*}$ & $4.48 \pm 0.25 \mathrm{AB}$ & $4.65 \pm 0.17 \mathrm{~A}$ \\
\hline $\mathrm{CK}+\mathrm{Si}$ & $4.38 \pm 0.26 \mathrm{a}$ & $4.14 \pm 0.26 \mathrm{a}$ & $3.25 \pm 0.17 \mathrm{a}$ & $2.59 \pm 0.03 \mathrm{ab}^{*}$ & $4.61 \pm 0.08 \mathrm{ab}$ & $4.66 \pm 0.40 \mathrm{a}$ \\
\hline $\mathrm{S}$ & $4.06 \pm 0.25 \mathrm{~A}^{*}$ & $3.75 \pm 0.24 \mathrm{AB}$ & $2.95 \pm 0.19 \mathrm{~B}$ & $3.47 \pm 0.18 \mathrm{~A}$ & $4.65 \pm 0.11 \mathrm{~A}$ & $4.80 \pm 0.12 \mathrm{~A}^{*}$ \\
\hline $\mathrm{S}+\mathrm{Si}$ & $4.56 \pm 0.07 \mathrm{a}^{*}$ & $3.930 .09 \mathrm{a}$ & $3.22 \pm 0.30 \mathrm{a}$ & $3.29 \pm 0.19 \mathrm{a}$ & $4.91 \pm 0.17 \mathrm{a}$ & $5.19 \pm 0.06 \mathrm{a}^{*}$ \\
\hline SD1 & $3.41 \pm 0.18 \mathrm{AB}^{*}$ & $3.43 \pm 0.10 \mathrm{~B}$ & $2.550 .13 \mathrm{~B}^{*}$ & $3.70 \pm 0.07 \mathrm{~A}^{*}$ & $4.29 \pm 0.08 \mathrm{AB}^{*}$ & $4.11 \pm 0.11 \mathrm{~B}^{*}$ \\
\hline $\mathrm{SD} 1+\mathrm{Si}$ & $3.55 \pm 0.07 \mathrm{a}^{*}$ & $3.670 .16 \mathrm{a}$ & $3.08 \pm 0.13 \mathrm{a}^{*}$ & $2.96 \pm 0.02 \mathrm{a}^{*}$ & $4.47 \pm 0.02 \mathrm{ab}^{*}$ & $4.40 \pm 0.11 \mathrm{a}^{*}$ \\
\hline SD2 & $3.13 \pm 0.14 \mathrm{~B}^{*}$ & $3.12 \pm 0.04 \mathrm{~B}^{*}$ & $2.76 \pm 0.08 \mathrm{~B}^{*}$ & $3.18 \pm 0.19 \mathrm{~A}^{*}$ & $4.03 \pm 0.04 \mathrm{~B}^{*}$ & $4.08 \pm 0.04 \mathrm{~B} *$ \\
\hline $\mathrm{SD} 2+\mathrm{Si}$ & $3.78 \pm 0.20 * \mathrm{a}$ & $3.55 \pm 0.16 \mathrm{a}^{*}$ & $3.21 \pm 0.13 \mathrm{a}^{*}$ & $2.06 \pm 0.00 \mathrm{~b}^{*}$ & $4.24 \pm 0.00 \mathrm{~b}^{*}$ & $4.34 \pm 0.07 \mathrm{a}^{*}$ \\
\hline
\end{tabular}


different depending on the treatments and development stages (Table 1). The Si addition significantly increased $\mathrm{Si}$ content in roost under all stressed conditions at 70 DAT and under SD2 at 110 DAT. Si addition significantly increased Si content in stems under SD1, SD2 at 70 DAT, while significantly decreased $\mathrm{Si}$ content in stem under CK, SD1, and SD2 at 110 DAT. However, the Si addition significantly increased $\mathrm{Si}$ content in leaves under all treatments except for S at 70 DAT.

Salinity combined with drought induced alterations in the anatomical structure of $G$. uralensis as compared to control. SD1 and SD2 increased the number of mesophyll cells and changed cell shape (long palisade cells were found under S, whereas palisade cells changed to foamy cells under SD1, SD2) (Fig. 1). Although some plastoglobuli were found in mesophyll cells under $\mathrm{S}$, the cells essentially appeared normal in leaves from control and $\mathrm{S}$ treatments (Fig. 2). However, under SD1 and SD2 it was observed that 1) the structure of chloroplast and mitochondria was badly damaged and contained numerous plastoglobuli; 2) the thylakoid network was incomplete, and the number of grana decreased as a result of thylakoid membranes degradation; and 3) the mitochondria became swollen, and their inner membranes were severely degraded (Fig. 2). However, addition of Si alleviated the structural changes of $G$. uralensis induced SD1 or SD2. Under the S stress, the cell arrangement of leaves was slightly loose, the number of capsule cells increased, and more of the stomata were formed near the lower epidermis by $\mathrm{Si}$ addition. Under SD1 and SD2 stress, the cells were arranged in a neat arrangement, foamy palisade cells were blocked, and there were more chloroplasts in palisade cells and spongy cells by Si addition (Fig. 1). Addition of Si maintained a well-preserved internal lamellar system in the chloroplasts under SD1 or SD2, and the chloroplasts contained less osmiophilic plastoglobuli and more starch grains (Fig. 2). They were still closely associated with the cell wall with few exceptions and showed an organized membrane system and most of the mitochondrial membrane structure was complete, with a few mitochondria membrane intact, but the ridge disappeared (Fig. 2).

Chlorophyll content in leaves was significantly affected by salinity combined with drought stress, developmental stages, and Si addition. Without Si addition, SD2 caused an increase in Chl $a$ and $\mathrm{Chl} a+b$ content, while a decrease in $\mathrm{Chl} b$ thus increase in Chl $a / b$ ratio compared with control at 70 DAT and at 110 DAT, SD1 caused a decrease in Chl $b$ content, whereas $\mathrm{Chl} a / b$ ratio significantly increased under SD1 and SD2 (Figs. 3 and 4). Under control condition, $\mathrm{Si}$ addition significantly increased $\mathrm{Chl} a$ content while decreased Chl $b$ content at 70 DAT. Under S stress, $\mathrm{Si}$ addition significantly increased $\mathrm{Chl} b$ and $\mathrm{Chl} a+b$

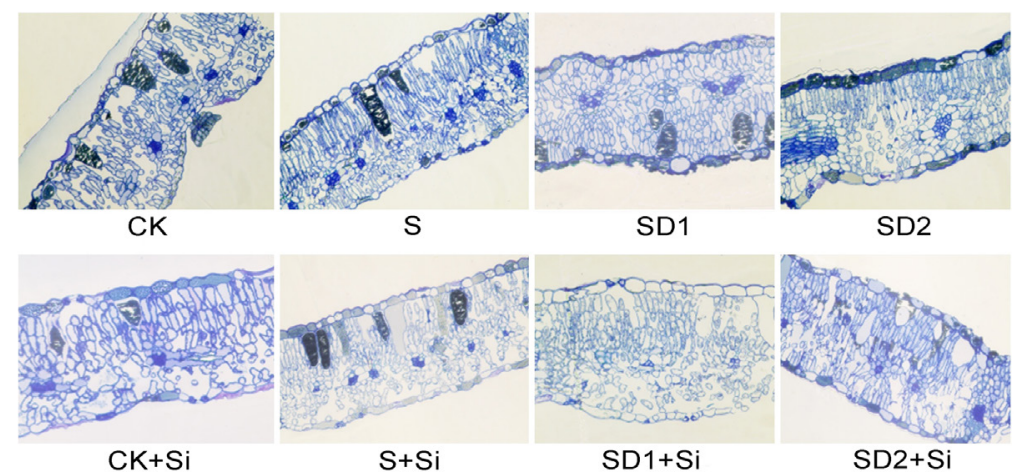

Fig. 1. Effect of $\mathrm{Si}\left[0.1 \mathrm{~g}\left(\mathrm{SiO}_{2}\right) \mathrm{kg}^{-1}\right.$ (dry soil) $]$ on microstructure (magnification 400) of Glycyrrhiza. uralensis leaves under control conditions (CK) salinity (S), and drought stresses $\left[6 \mathrm{~g}(\mathrm{NaCl}) \mathrm{kg}^{-1}(\right.$ soil $)+60-65 \%$ field water content $(\mathrm{FWC}), 6 \mathrm{~g}(\mathrm{NaCl}) \mathrm{kg}^{-1}(\mathrm{soil})+$ 45 - $50 \%$ FWC (SD1), $6 \mathrm{~g}(\mathrm{NaCl}) \mathrm{kg}^{-1}$ (soil) +30 - $35 \%$ FWC (SD2)].

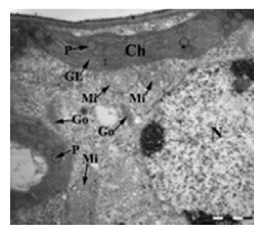

CK

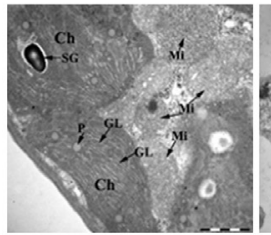

$\mathrm{CK}+\mathrm{Si}$

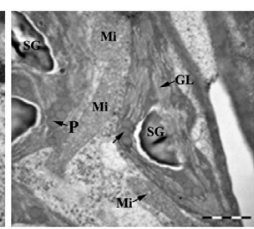

S

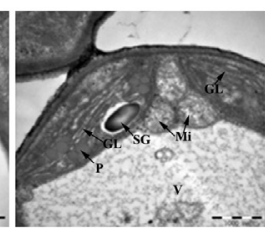

SD1

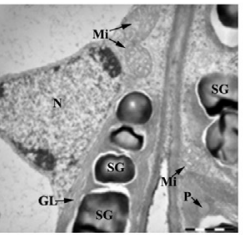

$\mathrm{S}+\mathrm{Si}$

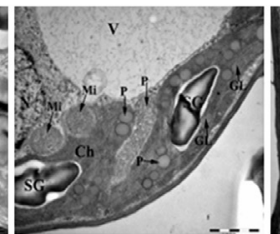

$\mathrm{SD} 1+\mathrm{Si}$

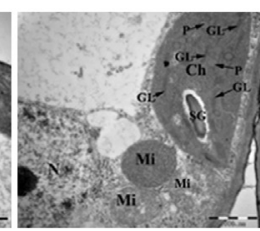

SD2

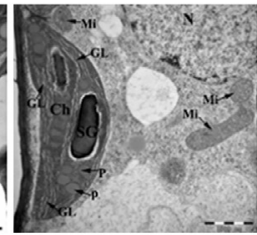

$\mathrm{SD} 2+\mathrm{Si}$

Fig. 2. Effect of Si on cellular ultrastructure of Glycyrrhiza uralensis leaves under control conditions (CK), salinity (S), and drought stress (SD1 and SD2) (for detail see Fig. 1) CW - cell wall, Ch - chloroplast, GL - thylakoid, Go - Golgi apparatus, Mi - mitochondrion, $\mathrm{N}$ - nucleus, $\mathrm{P}$ - plastoglobulus, $\mathrm{SG}$ - starch granule, V - vacuole (magnification 30000). 
at 110 DAT. Under SD1 stress, Si addition significantly decreased Chl $b$ and Chl $a+b$ content at 70 DAT and significantly increased $\mathrm{Chl} a+b$ content and $\mathrm{Chl} a / \mathrm{b}$ ratio at 110 DAT. Under SD2 stress, Si addition significantly increased Chl $a$, Chl $b$, and Chl $a+b$ content and Chl $a / b$ ratio except for $\mathrm{Chl} a / b$ ratio at 70 DAT (Figs. 3 and 4 ).

The gas exchange parameters $\left(\mathrm{E}, \mathrm{P}_{\mathrm{N}}, \mathrm{c}_{\mathrm{i}}\right.$, and $\left.\mathrm{g} s\right)$ gradually decreased with the increasing salt and drought

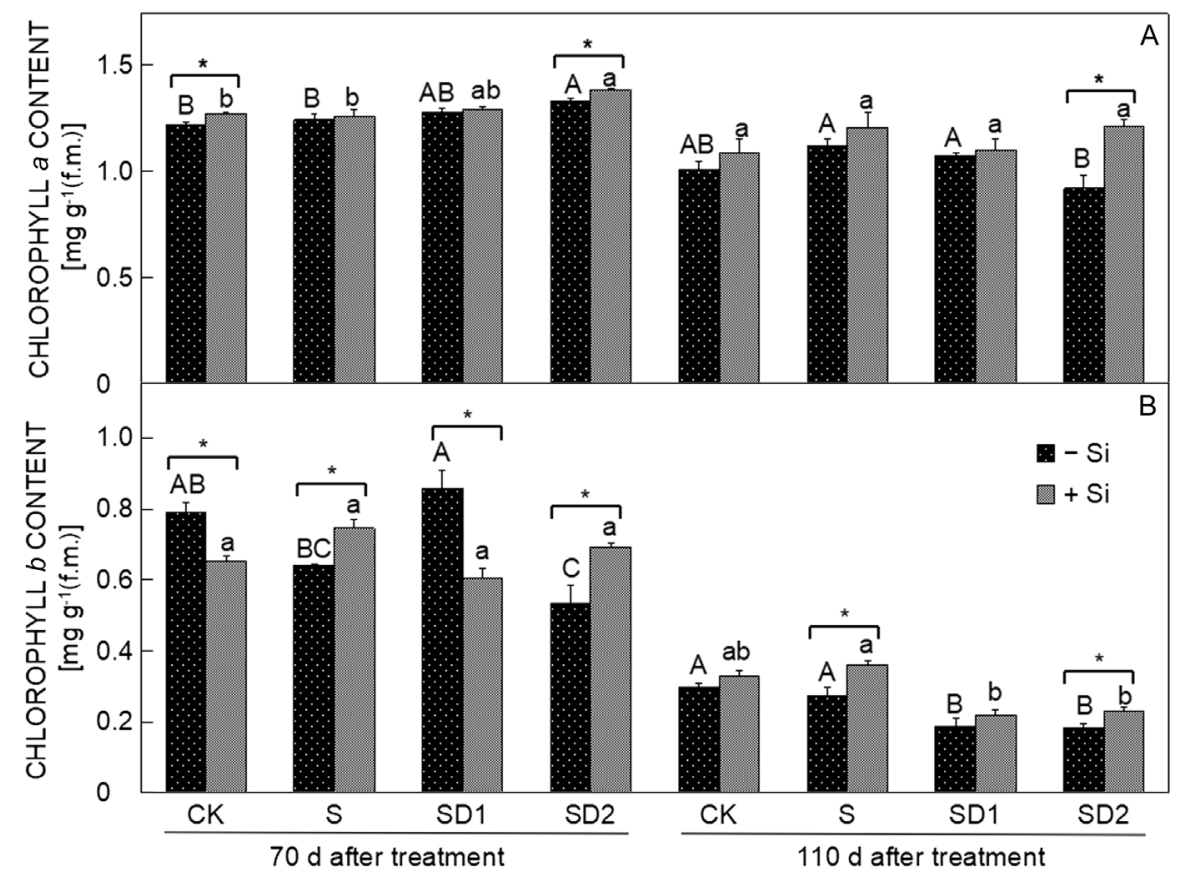

Fig. 3. Effect of Si on chlorophyll $a$ and chlorophyll $b$ content in Glycyrrhiza uralensis leaves under control conditions (CK), salinity (S), and drought stress (SD1 and SD2) (for detail see Fig. 1). Means \pm SEs, $n=6$, different letters within different treatments indicate significant differences at 0.05 probability; * stands for significant differences at 0.05 probability between two treatments with or without $\mathrm{Si}$.

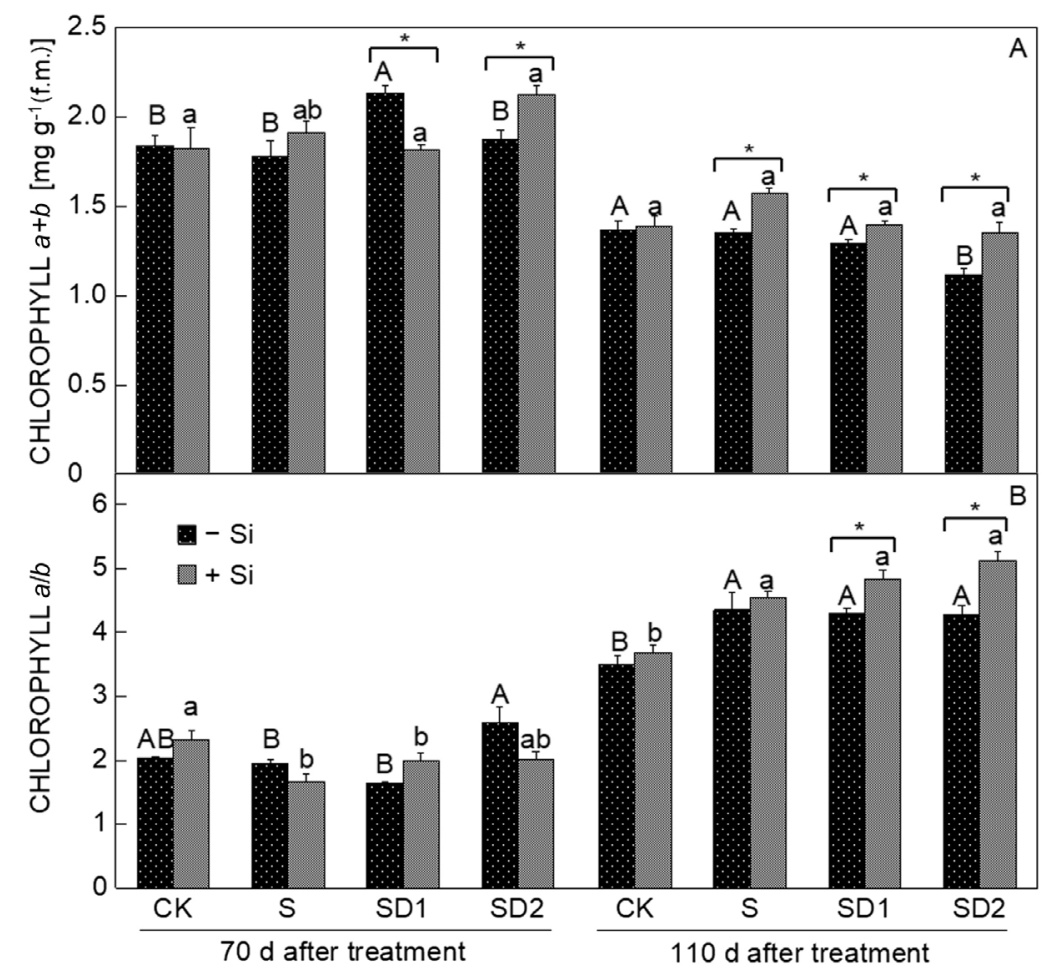

Fig. 4. Effect of Si on chlorophyll $a+b$ content and chlorophyll $a / b$ in Glycyrrhiza uralensis leaves under control conditions (CK), salinity (S), and drought stress (SD1 and SD2) (for detail see Fig. 1). Means \pm SEs, $n=6$, different letters within different treatments indicate significant differences at $\alpha=0.05 ;^{*}$ - significant differences at $\alpha=0.05$ between two treatments with or without $\mathrm{Si}$. 
Table 2. Effect of Si on growth characteristics of Glycyrrhiza uralensis plants under control, and salt and drought stresses. See Table 1 for detail.

\begin{tabular}{|c|c|c|c|c|c|c|}
\hline & \multicolumn{2}{|c|}{ Plant height $[\mathrm{cm}]$} & \multicolumn{2}{|l|}{ Root length $[\mathrm{cm}]$} & \multicolumn{2}{|c|}{ Root diameter [mm] } \\
\hline & 70 DAT & 110 DAT & $70 \mathrm{DAT}$ & 110 DAT & 70 DAT & 110 DAT \\
\hline CK & $44.96 \pm 0.53 \mathrm{~A}$ & $45.24 \pm 1.34 \mathrm{~A}$ & $34.70 \pm 1.03 \mathrm{~A}$ & $39.40 \pm 1.33 \mathrm{~A}$ & $9.04 \pm 0.20 \mathrm{~A}$ & $10.25 \pm 0.23 \mathrm{~A}$ \\
\hline $\mathrm{CK}+\mathrm{Si}$ & $45.12 \pm 0.42 \mathrm{a}$ & $49.32 \pm 1.65 \mathrm{a}$ & $35.76 \pm 1.50 \mathrm{a}$ & $38.79 \pm 2.04 \mathrm{a}$ & $9.07 \pm 0.20 \mathrm{a}$ & $10.75 \pm 0.18 \mathrm{a}$ \\
\hline $\mathrm{S}$ & $41.731 .73 \mathrm{~A}$ & $42.64 \pm 1.28 \mathrm{AB}^{*}$ & $30.50 \pm 0.19 \mathrm{~B}^{*}$ & $33.98 \pm 0.89 \mathrm{~B}$ & $8.66 \pm 0.32 \mathrm{~A}$ & $9.16 \pm 0.14 \mathrm{~B}^{*}$ \\
\hline $\mathrm{S}+\mathrm{Si}$ & $44.79 \pm 0.62 \mathrm{a}$ & $47.29 \pm 1.07 \mathrm{a}^{*}$ & $34.21 \pm 0.085 \mathrm{a}^{*}$ & $35.36 \pm 1.21 \mathrm{ab}$ & $9.05 \pm 0.65 a$ & $10.56 \pm 0.55 \mathrm{a}^{*}$ \\
\hline SD1 & $32.36 \pm 1.61 \mathrm{~B}^{*}$ & $40.541 .19 \mathrm{~B}$ & $25.09 \pm 1.84 \mathrm{C}$ & $32.09 \pm 0.92 \mathrm{~B}$ & $8.03 \pm 0.45 \mathrm{~A}$ & $8.77 \pm 0.29 \mathrm{AB}^{*}$ \\
\hline $\mathrm{SD} 1+\mathrm{Si}$ & $38.66 \pm 1.83 b^{*}$ & $40.29 \pm 0.12 b$ & $26.550 .70 b$ & $34.30 \pm 0.94 \mathrm{ab}$ & $8.43 \pm 0.08 \mathrm{a}$ & $9.20 \pm 0.44 b^{*}$ \\
\hline $\mathrm{SD} 2$ & $32.74 \pm 0.14 \mathrm{~B}^{*}$ & $34.410 .77 \mathrm{C}$ & $13.00 \pm 0.20 \mathrm{D}^{*}$ & $30.44 \pm 0.94 \mathrm{~B}$ & $7.82 \pm 0.19 \mathrm{~A}^{*}$ & $7.97 \pm 0.33 C^{*}$ \\
\hline \multirow[t]{3}{*}{$\mathrm{SD} 2+\mathrm{Si}$} & $37.63 \pm 1.54 b^{*}$ & $36.12 \pm 1.82 \mathrm{a}$ & $16.470 .64 \mathrm{c}^{*}$ & $32.22 \pm 1.12 b$ & $8.47 \pm 0.28 \mathrm{a}^{*}$ & $8.76 \pm 0.07 b^{*}$ \\
\hline & \multicolumn{2}{|l|}{ Leaf area $\left[\mathrm{mm}^{2}\right]$} & \multicolumn{2}{|c|}{ Stem diameter $[\mathrm{mm}]$} & & \\
\hline & 70 DAT & 110 DAT & $70 \mathrm{DAT}$ & 110 DAT & & \\
\hline $\mathrm{CK}$ & $8.28 \quad 0.23 \mathrm{~A}$ & $9.89 \pm 0.78 \mathrm{~A}$ & $2.46 \pm 0.065 \mathrm{~A}$ & $2.31 \pm 0.03 \mathrm{~A}^{*}$ & & \\
\hline $\mathrm{CK}+\mathrm{Si}$ & $7.75 \pm 0.57 \mathrm{a}$ & $10.43 \pm 1.00 \mathrm{a}$ & $2.56 \pm 0.52 \mathrm{ab}$ & $2.54 \pm 0.08 \mathrm{a}^{*}$ & & \\
\hline $\mathrm{S}$ & $4.94 \pm 0.44 \mathrm{~B}$ & $9.02 \pm 0.24 \mathrm{AB}^{*}$ & $2.36 \pm 0.04 \mathrm{~A}^{*}$ & $2.19 \pm 0.08 \mathrm{AB}$ & & \\
\hline $\mathrm{S}+\mathrm{Si}$ & $6.03 \pm 0.25 \mathrm{a}$ & $10.34 \pm 0.22 \mathrm{a}^{*}$ & $2.650 .09 \mathrm{a}^{*}$ & $2.32 \pm 0.17 \mathrm{a}$ & & \\
\hline SD1 & $3.77 \pm 0.29 \mathrm{~B}^{*}$ & $8.64 \pm 0.37 \mathrm{AB}^{*}$ & $2.040 .04 \mathrm{~B}^{*}$ & $2.10 \pm 0.01 \mathrm{~B}^{*}$ & & \\
\hline $\mathrm{SD} 1+\mathrm{Si}$ & $6.17 \pm 0.72 \mathrm{a}^{*}$ & $10.05 \pm 0.29 \mathrm{ab} *$ & $2.370 .04 b^{*}$ & $2.44 \pm 0.09 \mathrm{a}^{*}$ & & \\
\hline $\mathrm{SD} 2$ & $4.62 \pm 0.46 \mathrm{~B}^{*}$ & $7.24 \pm 0.24 \mathrm{~B}^{*}$ & $2.110 .01 * \mathrm{~B}$ & $2.1 \pm \pm .11 \mathrm{~B}$ & & \\
\hline $\mathrm{SD} 2+\mathrm{Si}$ & $6.62 \pm 0.66 \mathrm{a}^{*}$ & $7.970 .27 \mathrm{~b}^{*}$ & $2.31 \pm 0.07 b^{*}$ & $2.24 \pm 0.03 \mathrm{a}$ & & \\
\hline
\end{tabular}

Table 3. Effect of Si on leaf dry mass, stem dry mass, root dry mass, and total dry mass of Glycyrrhiza uralensis plants under control, and salt and drought stresses. See Table 1 for detail.

\begin{tabular}{|c|c|c|c|c|}
\hline & \multicolumn{2}{|c|}{ Leaf dry biomass $\left[\mathrm{g} \mathrm{pot}^{-1}\right]$} & \multicolumn{2}{|c|}{ Stem dry biomass $\left[\mathrm{g} \mathrm{pot}^{-1}\right]$} \\
\hline & 70 DAT & 110 DAT & 70 DAT & 110 DAT \\
\hline $\mathrm{CK}$ & $11.00 \pm 1.11 \mathrm{~A}$ & $7.93 \pm 1.51 \mathrm{~A}$ & $9.24 \pm 0.05 \mathrm{~A}$ & $11.31 \pm 0.05 \mathrm{~A}^{*}$ \\
\hline $\mathrm{CK}+\mathrm{Si}$ & $10.69 \pm 1.15 b$ & $8.65 \pm 0.56 a$ & $10.22 \pm 0.27 b$ & $13.10 \pm 0.84 \mathrm{a}^{*}$ \\
\hline S & $12.01 \pm 0.62 \mathrm{~A}$ & $5.11 \pm 0.14 \mathrm{~B}^{*}$ & $9.00 \pm 0.34 \mathrm{~A}^{*}$ & $9.04 \pm 0.61 \mathrm{~B}^{*}$ \\
\hline $\mathrm{S}+\mathrm{Si}$ & $11.97 \pm 1.68 \mathrm{a}$ & $5.94 \pm 0.09 b^{*}$ & $11.24 \pm 0.12 \mathrm{a}^{*}$ & $11.35 \pm 0.68 \mathrm{a}^{*}$ \\
\hline SD1 & $6.58 \pm 0.19 \mathrm{~B}^{*}$ & $4.84 \pm 0.11 \mathrm{~B}$ & $6.11 \pm 0.67 \mathrm{~B}$ & $5.95 \pm 0.36 \mathrm{C}$ \\
\hline $\mathrm{SD} 1+\mathrm{Si}$ & $7.70 \pm 0.18 \mathrm{c}^{*}$ & $4.62 \pm 0.53 b$ & $6.63 \pm 0.13 c$ & $6.41 \pm 0.67 b$ \\
\hline $\mathrm{SD} 2$ & $3.29 \pm 0.49 C^{*}$ & $1.14 \pm 0.03 \mathrm{C}$ & $3.96 \pm 0.28 \mathrm{C}^{*}$ & $4.05 \pm 0.14 \mathrm{D} *$ \\
\hline \multirow[t]{3}{*}{$\mathrm{SD} 2+\mathrm{Si}$} & $6.36 \pm 0.49 c^{*}$ & $1.38 \pm 0.15 \mathrm{c}$ & $6.08 \pm 0.55 c^{*}$ & $4.74 \pm 0.21 b^{*}$ \\
\hline & \multicolumn{2}{|c|}{ Root dry mass $\left[\mathrm{g} \mathrm{pot}^{-1}\right]$} & \multicolumn{2}{|c|}{ Total dry mass $\left[\mathrm{g} \mathrm{pot}^{-1}\right]$} \\
\hline & 70 DAT & $110 \mathrm{DAT}$ & 70 DAT & 110 DAT \\
\hline CK & $34.70 \pm 1.03 \mathrm{~A}$ & $58.97 \pm 5.02 \mathrm{~A}$ & $54.94 \pm 2.19 \mathrm{~A}$ & $78.21 \pm 6.58 \mathrm{~A}$ \\
\hline $\mathrm{CK}+\mathrm{Si}$ & $35.76 \pm 1.50 \mathrm{a}$ & $57.35 \pm 1.95 \mathrm{a}$ & $56.67 \pm 2.92 \mathrm{a}$ & $79.09 \pm 3.34 \mathrm{a}$ \\
\hline $\mathrm{S}$ & $30.50 \pm 0.19 \mathrm{~B}^{*}$ & $52.311 .05 \mathrm{AB}$ & $51.51 \pm 1.16 \mathrm{~A}^{*}$ & $66.45 \pm 1.79 \mathrm{~B}$ \\
\hline $\mathrm{S}+\mathrm{Si}$ & $34.21 \pm 0.09 \mathrm{a}^{*}$ & $53.65 \pm 1.26 \mathrm{a}$ & $57.421 .88 \mathrm{a}^{*}$ & $70.94 \pm 2.33 b$ \\
\hline SD1 & $25.09 \pm 0.36 \mathrm{C}^{*}$ & $46.08 \pm 0.44 \mathrm{~B}^{*}$ & $37.781 .22 \mathrm{~B}$ & $56.87 \pm 0.91 \mathrm{C}$ \\
\hline $\mathrm{SD} 1+\mathrm{Si}$ & $26.55 \pm 0.70 \mathrm{~b}^{*}$ & $48.36 \pm 0.36 b^{*}$ & $40.88 \pm 1.01 \mathrm{~b}$ & $59.38 \pm 1.57 \mathrm{c}$ \\
\hline SD2 & $13.04 \pm 0.25 \mathrm{D}^{*}$ & $27.03 \pm 0.19 C^{*}$ & $20.291 .01 C^{*}$ & $32.22 \pm 0.35 \mathrm{D}^{*}$ \\
\hline $\mathrm{SD} 2+\mathrm{Si}$ & $16.47 \pm 0.64 b^{*}$ & $30.370 .13 c^{*}$ & $28.91 \pm 1.68 \mathrm{c}^{*}$ & $36.38 \pm 0.48 \mathrm{~d}^{*}$ \\
\hline
\end{tabular}

stress except for $\mathrm{c}_{\mathrm{i}}$ which only significantly decreased under SD2 (Fig. 4). Si addition significantly increased gas exchange parameters under all stress conditions except for $\mathrm{c}_{\mathrm{i}}$ under S stress (Fig. 5). The RWC of G. uralensis leaves significantly decreased under SD1 and SD2 compared with control and reached the least value under 
SD2 (Fig. 6). The WUE significantly decreased under SD2 (Fig. 7). However, the $\mathrm{Si}$ addition could maintain better water status (higher RWC) and increased WUE under all stresses and especially under SD1 and SD2 (Figs. 6 and 7). The Si content in stems positively correlated with $\mathrm{E}, \mathrm{P}_{\mathrm{N}}, \mathrm{c}_{\mathrm{i}}$, and RWC, while Si content in leaves positively correlated with $g_{s}$ and WUE (Table 1 Suppl.).

Morphological parameters (root length, root diameter, plant height, stem diameter, and leaf area) and the dry matter accumulation (leaf dry mass, stem dry mass, root dry mass, and total dry mass) were reduced in combined stress when compared with control and reached minimum value under SD2 (Tables 2 and 3). The Si addition markedly changed the growth of $G$. uralensis plants. Especially under $\mathrm{S}$ stress, the $\mathrm{Si}$ addition significantly increased root length, stem diameter, and root and total dry mass at 70 DAT, as well as significantly increased root diameter, leaf area, and leaf dry mass at 110 DAT. Under SD1 stress, the $\mathrm{Si}$ addition significantly increased plant height and leaf dry mass at 70 DAT and significantly increased root length and root diameter at 110 DAT, while it significantly increased stem diameter, leaf area and root dry massat both 70 and 110 DAT. Under SD2 stress, the Si addition significantly increased all morphological parameters at 70 DAT, but root diameter and leaf area also at 110 DAT. The dry matter accumulation was significantly increased by $\mathrm{Si}$ addition during the experiment except for leaf dry mass which only increased at 70 DAT (Tables 2 and 3). In this study, we also found a positive correlation between growth parameters, photosynthesis, and water status. Specifically, leaf dry mass and leaf area were positive correlation with $\mathrm{E}, \mathrm{P}_{\mathrm{N}}, \mathrm{c}_{\mathrm{i}}, \mathrm{g}_{\mathrm{s}}$, and WUE. Stem dry mass was positive correlation with gas exchange parameters and RWC. The root dry mass positively correlation with $\mathrm{P}_{\mathrm{N}}, \mathrm{g}_{\mathrm{s}}$,

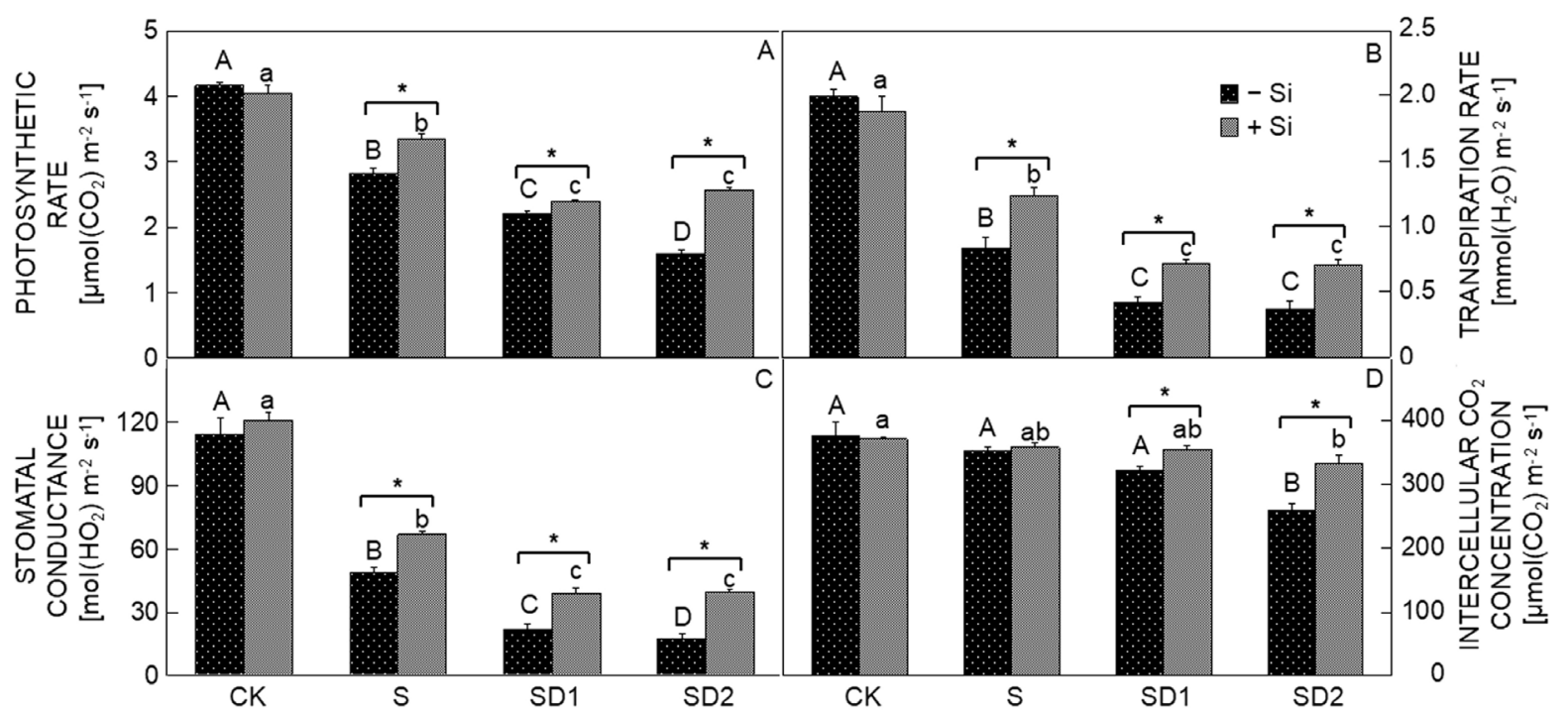

Fig. 5. Effect of $\mathrm{Si}$ on net photosynthetic rate $(A)$, transpiration rate $(B)$, stomatal conductance $(C)$, and intercellular $\mathrm{CO}_{2}$ concentration (D) of Glycyrrhiza uralensis leaves under control conditions (CK), salinity (S), and drought stress (SD1 and SD2) (for detail see Fig. 1) Means \pm SEs, $n=6$, different letters within different treatments indicate significant differences at 0.05 probability; * stands for significant differences at 0.05 probability between two treatments with or without $\mathrm{Si}$.

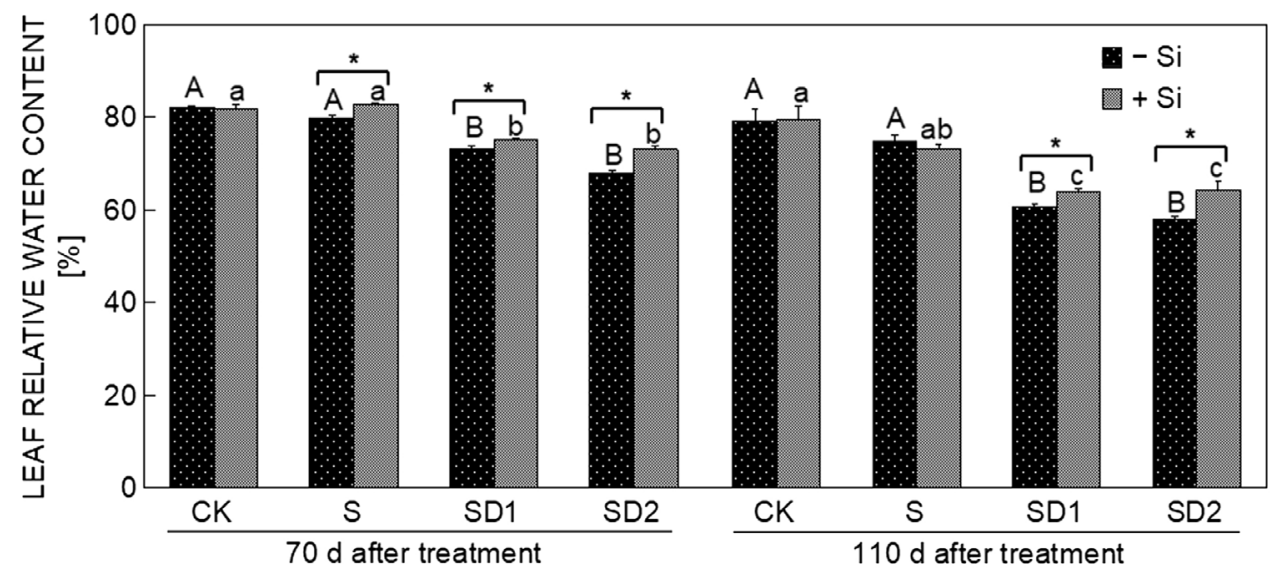

Fig. 6. Effect of Si on relative water content of Glycyrrhiza uralensis leaves under control conditions (CK), salinity (S), and drought stress (SD1 and SD2) (for detail see Fig. 1) at 70 and 110 days after treatment. Means \pm SEs, $n=6$, different letters within different treatments indicate significant differences at 0.05 probability; ${ }^{*}$ stands for significant differences at 0.05 probability between two treatments with or without Si. 
WUE, and RWC and total dry omass positively correlated with $\mathrm{E}, \mathrm{P}_{\mathrm{N}}, \mathrm{c}_{\mathrm{i}}$, and WUE (Table 2 Suppl.).

\section{Discussion}

All plants grown in soil contain $\mathrm{Si}$ in their tissues, and Si content in plants varies greatly among species and genotypes (Ma and Yamaji 2008). G. uralensis was weak in Si absorption rate, but $G$. uralensis plants can accumulate a certain amount of $\mathrm{Si}$ with the extension of the absorption time (Zhang et al. 2017). It is commonly accepted that $\mathrm{Si}$ is absorbed by plant root in the form of soluble silicic acid. After being absorbed, silicic acid is then transported to the shoot via xylem (Ma and Yamaji 2008). Silicic acid finally polymerizes and precipitates, forming "opal phytoliths" in the cell wall, intercellular spaces, and trichomes (Cooke and Leishman 2011, Mazumdar 2011). A significant decrease of Si content in tomato roots was observed under salt stress by Muneer and Jeong (2015). In this study, Si content in $G$. uralensis roots and leaves was decreased by SD1, SD2 treatments at 70 and 110 DAT and Si content in stem only at 70 DAT (Table 1). These results indicated that these stresses decreased $\mathrm{Si}$ absorption by roots and further inhibited transportation to shoot via xylem. Si addition resulted in markedly increased $\mathrm{Si}$ content in roots and leaves while decreased it in stems under SD1 and SD2 at 110 DAT (Table 3), which was similar to the previous studies in soybean (Li et al. 2004), tomato (Cao et al. 2015), and wheat (Ma et al. 2016). The higher content of Si under SD1 and SD2 in plants roots and leaves might be due to deposition of $\mathrm{Si}$ in cell walls and endodermis, which can reduce the translocation of $\mathrm{NaCl}$ (Liang et al. 2005). The Si applied also could strengthen the membranes of plant cells, change their permeability, and maintain the integrity of cellular structures (Figs. 1 and 2). In addition, Ma et al. (2016) find that application of Si in the field can alleviate damage of photosynthesis induced by drought stress in wheat. This study further confirms that Si content in stem and leaf of G. uralensis was positively correlated with photosynthetic parameters and water status (Table 1 Suppl.). These results indicated that $\mathrm{Si}$ addition enhanced

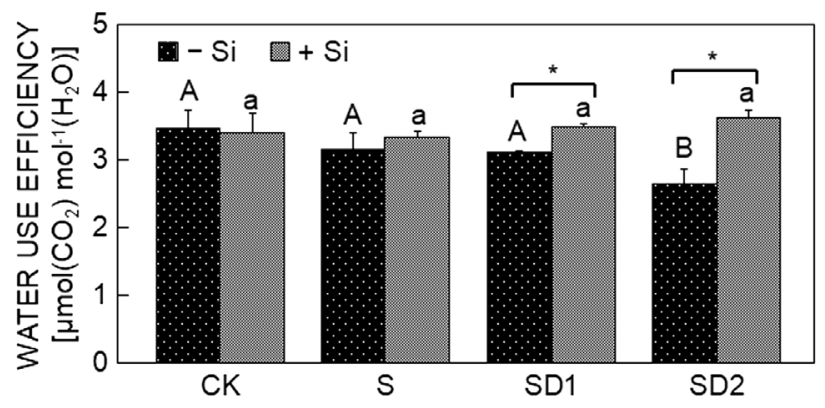

Fig. 7. Effect of Si on water use efficiency of Glycyrrhiza uralensis leaves under control conditions (CK), salinity (S), and drought stress (SD1 and SD2) (for detail see Fig. 1) at 70 and 110 days after treatment. Means \pm SEs, $n=6$, different letters within different treatments indicate zignificant differences at 0.05 probability; * stands for significant differences at 0.05 probability between two treatments with or without $\mathrm{Si}$. uptake, transport and accumulation of $\mathrm{Si}$, which played a significant role in improving the growth and dry matter accumulation of G. uralensis plants. Plant leaves are very sensitive to environmental changes and salt and drought stresses significantly changed leaf structure: the mesophyll cells became smaller and irregular in shape, with more influence on spongy tissue than on palisade tissues. The addition of $\mathrm{Si}$ inhibited the degeneration of membrane systems, mesophyll cells were lined regularly, and vascular area increased slightly (Fig. 1). In this process, palisade tissue cells contain more larger chloroplasts arranged relatively close, which can greatly improve the photosynthetic rate of plants, but can also reduce the evaporation of water (Keremu and Hou 2006). Some studies have found that the more developed main vascular bundle, the higher water transport efficiency. Therefore, the developed vascular bundle structure may ensure water and nutrient transport and has the function of water conservation and storage (He et al. 2005). The previous results showed that water deficit and heat stress in rice caused deformed mesophyll cells, but some of the cells could maintain the normal shape by $\mathrm{Si}$ addition (Agarie et al. 1998), which was similar to the present results.

Chloroplast is the first organelle of mesophyll cells which could be damaged during the salt and drought stresses (Noode'n et al. 2004). Generally, salt- and drought-induced lipid peroxidation results in a massive reorganization of leaf chloroplast ultrastructure (Bejaoui et al. 2016), which is considered to be a good indicator of plant status under stress conditions ( $\mathrm{Xu}$ et al. 2009). Previous study found that the number of chloroplast and intercellular space decreased dramatically, and the disintegrated chloroplast envelope together with the loosen and swollen grana thylakoids can be observed under salt and drought stressed G. uralensis seedlings (Liu et al. 2010), these changes are similar to that observed in the present study under salt combined with drought stress (Fig. 2). It has been reported that the number and size of plastoglobuli increases due to thylakoid membranes degeneration (Olmos et al. 2007), which was corresponded with more plastoglobuli found under SD2 than that under SD1 (Fig. 2). And the disorganization of thylakoid membranes is correlated to the $\mathrm{H}_{2} \mathrm{O}_{2}$ accumulation in chloroplasts (Oksanen et al. 2005). In addition, changed shape of many chloroplasts from lenticular to round or oval under SD1 and SD2 (Fig. 2) suggests an altered function of them. Also Zellnig et al. (2004) observed that stress-impaired growth in leaves of drought-sensitive spinach lines is accompanied with the reduced length and abnormal shape of chloroplasts. Interestingly, it was evident that $\mathrm{Si}$ content in leaves of G. uralensis increased by $\mathrm{Si}$ addition (Table 1), which would be deposited on cell walls (Liang et al. 2005). And Si applied could strengthen the membranes of plant cells by depositing around the cell walls and changing their permeability under SD1 and SD2 (Fig. 2). As the light reactions of photosynthesis occur in the grana, a close relationship is expected between thylakoid membrane integrity and photosynthetic activity (Chen et al. 2011a). Also, the mitochondria were intact and possessed electrondense bodies after Si addition which would improve the 
energy metabolism of $G$. uralensis under drought and salt stresses. In addition, more and larger starch grains after $\mathrm{Si}$ addition were observed in the present study. These results suggested that $\mathrm{Si}$ application helped to maintain the ultrastructure of $G$. uralensis leaves, which enabled a normal physiological functions of the plant exposed to salt combined with drought stress.

The decline in Chl content under salt or drought has been considered to be a typical symptom of pigment photooxidation and chlorophyll degradation (Hajihashemi and Ehsanpour 2013). Ping et al. (2015) observed a reduction in $\mathrm{Chl}$ content in apple under progressive drought stress. Decreased or unchanged Chl content during drought stress has been reported in some species, depending on the duration and severity of drought (Silva et al. 2012). In our study, Chl $a$ content increased under SD2 at 70 DAT while Chl $b$ content decreased under SD1 and SD2 at 110 DAT. Correspondingly, Chl $a / b$ significantly increased under SD1 and SD2 at 110 DAT (Fig. 3), indicating that $\mathrm{Chl} b$ was more sensitive than Chl $a$ to stress similarly as found Sultana et al. (1999). In addition, Chl $a+b$ content increased under SD1 at 70 DAT while declined under SD2 at 110 DAT. The Si application markedly affected chlorophyll content in $G$. uralensis leaves in a stress degrees and developmental stage dependent manner. Generally, at 70 DAT, Si addition increased Chl $a$ content under control and SD2 but decreased Chl $b$ under control and SD1. At 110 DAT, Si addition increased Chl_ $a$ and $\mathrm{Chl} a+b$ content under SD2 and $\mathrm{Chl} b$ under S and $\mathrm{SD} 2$. This indicates that in the course of stress time, $\mathrm{Si}$ addition increased Chl content mainly under SD2 and thus increased Chl $a / b$ under SD1 and SD2. These results are similar to Si-induced increase in $\mathrm{Chl} a$ and $\mathrm{Chl} b$ content under drought stress (Maghsoudi et al. 2016). Combined with the above results, Si could maintain the structural integrity of mesophyll cells and chloroplasts to a certain extent and increase the amount of chloroplasts (Figs 1, 2, and 3 ).

The influence of drought stress is usually perceived as a decline in photosynthetic rate. When the amount of available soil water is moderately or severely limiting, the first response of plants is to close their stomata to minimize water loss, and decreases the influx of $\mathrm{CO}_{2}$ into the leaves. This is accompanied by decreases in $\mathrm{g}_{\mathrm{s}}$, $\mathrm{E}$, and $\mathrm{P}_{\mathrm{N}}$. In this study, $\mathrm{E}, \mathrm{P}_{\mathrm{N}}$, and $\mathrm{g}_{\mathrm{s}}$ were concomitantly decreased under salinity combined with drought stress (Fig. 5). These results suggested that the suppression of $\mathrm{P}_{\mathrm{N}}$ could be generally attributed to stomatal limitation rather than non-stomatal limitation (Zhang et al. 2013). These findings are in agreement with previous studies in melon and rice (Huang et al. 2011, Porcel et al. 2015). The application of Si reduced the impact of these stresses which was manifested by improved gas exchange parameters. A similar effect of Si was documented for salt stressed tomato (Haghighi and Pessarakli 2013) and cotton (Farooq et al. 2013). In the present study, Si significantly elevated $\mathrm{P}_{\mathrm{N}}$ under all stressed conditions. This could have been due to increase in leaf area, Chl content, and number and size of stomata (Fig. 1 and 2). In addition, this finding was similar to those of Da-Cunha and Do-
Nascimento (2009) who studied cadmium stressed maize plants and found that $\mathrm{Si}$ resurrected the photosynthetic potential due to improved integrity of the photosynthetic apparatus (Fig. 1 and 2). The $\mathrm{c}_{\mathrm{i}}$ can be used to discriminate between changes in $\mathrm{P}_{\mathrm{N}}$ resulting from stomatal limitation or non-stomatal limitations. From our results, the increase in $\mathrm{P}_{\mathrm{N}}$ caused by $\mathrm{Si}$ addition maybe resulted from stomatal limitations because the $\mathrm{Si}$ application significantly enhanced the $g_{s}$ under SD1 and SD2 (Fig. 5) which could be associated with the increase in the number of stomata and stomatal size (Fig. 1 and 2). The positive impacts of $\mathrm{Si}$ on plants under stress conditions were often associated with transpiration reduction (Gao et al. 2006). However, our study showed that $\mathrm{E}$ in leaves of stressed G. uralensis was significantly increased by $\mathrm{Si}$ addition (Fig. 5). The similar phenomenon was observed in sorghum (Hattori et al. 2005) and rice (Chen et al. 2011b) under drought stress. Also Sonobe et al. (2011) investigated the diurnal changes of gas exchange parameters in sorghum grown hydroponically and found that $\mathrm{Si}$ induces higher $\mathrm{g}_{\mathrm{s}}, \mathrm{P}_{\mathrm{N}}$, and $\mathrm{E}$ than in untreated plants under water stress.

The RWC has been reported as a good indicator of salt and drought stresses tolerance (Kraus et al. 1995). The WUE for plants is very important, especially when they are subjected to drought. The salinity and drought can reduce WUE in wheat (Gong and Chen 2012) and Sorgum bicolor (Yin et al. 2013). In the present study, RWC was declined under both SD1 and SD2. This result also in accordance with previous results in tobacco (Dobra et al. 2010). In addition, a significant decrease of WUE was observed under SD2, while significantly improvement was observed after Si treatment. Similarly, Si improves the water status of drought stressed wheat (Ding et al. 2007) and drought and UV-B radiation stressed soybean seedlings (Shen et al. 2010). In the present study, the enhanced WUE in Si-treated stressed plants indicates an augmentation in carbon assimilation rate, which resulted in better plant growth under stressed conditions (Fernandez-Garcia et al. 2014). Our results also indicated that $\mathrm{Si}$ increased RWC of $G$. uralensis, which is in agreement with the findings of Shen et al. (2010) and the improvement of growth could have also been due to this fact. It is suggested that Si could decrease cuticular and stomatal transpiration (Gao et al. 2006). However, Liang et al. (2008) proposed that accumulation of polar monosilicic acid and/or polymerized silicic acid in epidermal cell walls may form hydrogen bonds between water and hydrated silica, therefore, making water molecules less easily to escape from leaf surface. In the present study, both $\mathrm{g}_{\mathrm{s}}$ and $\mathrm{E}$ of G. uralensis leaves increased by $\mathrm{Si}$ application under salinity combined with drought stress, indicating that $\mathrm{Si}$ might regulate root water uptake and transport in response to water loss and maintain water balance in $G$. uralensis plants under stress conditions. Which can be confirmed by improvement of RWC. These results are in accordance with previous findings in sorghum (Hattori et al. 2005) and pepper (Lobato et al. 2009).

The most common and significant effect of salt and drought stresses on plants is the inhibition of growth and biomass production (Kavas et al. 2015). Our results 
showed that SD1 and SD2 decreased G. uralensis growth and biomass compared with control, and this adverse effect was partly reversed by Si addition (Table 2 and 3), which is consistent with previous findings in sugarcane (Ashraf et al. 2010), G. uralensis seedlings (Zhang et al. 2017), and canola (Hashemi et al. 2010). The salinity alone had no significant effect on growth and biomass of $G$. uralensis plants compared with control (Table 2 and 3), indicating that G. uralensis is resistant to moderate salt stress (Yang et al. 2006). We also found that the benificial effect of Si on growth and biomass was strongest SD2 at 70 DAT. Furthermore, we also found a strong positive correlation between growth parameters and photosynthesis parameters and water status (Table 2 Suppl.).

Based on the results of this study, it could be concluded that under salinity combined with drought, Si application resulted in markedly increased of Si content in leaves due to its deposition on cell walls. The Si application helped to maintain the integrity of anatomical structure, to improve Chl content, and to increase $\mathrm{P}_{\mathrm{N}}, \mathrm{E}, \mathrm{g}_{\mathrm{s}}$ and WUE. All of the above results ultimately led to higher growth and biomass production of $G$. uralensis plants under salinity combined with drought stress after Si application.

\section{References}

Abbas, T., Balal, R.M., Shahid, M.A., Pervez, M.A., Ayyub, C.M., Aqueel, M.A., Muhammad Javaid, M.M.: Siliconinduced alleviation of $\mathrm{NaCl}$ toxicity in okra (Abelmoschus esculentus) is associated with enhanced photosynthesis, osmoprotectants and antioxidant metabolism. - Acta Physiol. Plant. 37: 1-15, 2015.

Agarie, S., Hanaoka, N., Ueno, O., Miyazaki, A., Kubota, F., Agata, W., Peter, B.K.: Effects of silicon on tolerance to water deficit and heat stress in rice plants (Oryza Sativa L.), monitored by electrolyte leakage. - Plant Prod. Sci. 1: 96-103, 1998.

Aminian, R., Mohammadi, S., Hoshmand, S., Khodombashi, M.: Chromosomal analysis of photosynthesis rate and stomatal conductance and their relationships with grain yield in wheat (Triticum aestivum L.) under water-stressed and well-watered conditions. - Acta Physiol. Plant. 33: 755-764, 2011.

Ashraf, M., Rahmatullah, A.R., Bhatti, A.S., Afzal, M., Sarwar, A., Maqsood, M.A., Kanwa, S.: Amelioration of salt stress in sugarcane (Sacharum officinarum L.) by supplying potassium and silicon in hydroponics. - Pedosphere 20: 153-162, 2010.

Bejaoui, F., Salas, J.J., Nouairi, I., Smaoui, A., Abdelly, C., Martínez-Force, E., Youssef, N.B.: Changes in chloroplast lipid contents and chloroplast ultrastructure in Sulla carnosa and Sulla coronaria leaves under salt stress - J. Plant Physiol. 198: $32-38,2016$

Bijanzadeh, E., Emam, Y.: Effect of source-sink manipulation on yield components and photosynthetic characteristic of wheat cultivars (Triticum aestivum and T. durum L.). - J. appl. Sci. 10: $564-569,2010$.

Cao, B.L., Ma, Q., Zhao, Q., Wang, L., Xu, K.: Effects of silicon on absorbed light allocation, antioxidant enzymes and ultrastructure of chloroplasts in tomato leaves under simulated drought stress. - Sci. Hort. 194: 53-62, 2015.

Chen, J., Wu, F.H., Wang, W.H., Zheng, C.J., Lin, G.H., Dong, X.J., He, X.J., Pei, Z.M., Zheng, H.L.: Hydrogen sulphide enhances photosynthesis through promoting chloroplast biogenesis, photosynthetic enzyme expression, and thiol redox modification in Spinacia oleracea seedlings. - J. exp. Bot. 62: 4481-4493, 2011a.

Chen, W., Yao, X., Cai, K., Chen, J.: Silicon alleviates drought stress of rice plants by improving plant water status, photosynthesis and mineral nutrient absorption. - Biol. Trace Element Res. - 142: 67-76, $2011 \mathrm{~b}$.

Cooke, J., Leishman, M.R.: Is plant ecology more siliceous than we realise? - Trends Plant Sci. 16: 61-68, 2011.

Da-Cunha, K.P.V., Do-Nascimento, C.W.A.: Silicon effects on metal tolerance and structural changes in maize (Zea mays) grown on cadmium and zinc enriched soil. - Water Air Soil Pollut. 197: 323-330, 2009.

Ding, Y.F., Liang, Y.C., Zhu, J., Li, Z.J.: Effects of Si on plant growth, photosynthetic parameters and soluble sugar content in leaves of wheat under drought stress. - Plant Nutr. Fert. Sci. 13: 471-478, 2007.

Dobra, J., Motyka, V., Dobrev, P., Malbeck, J., Prasil, I. T., Haisel, D., Gaudinova, A., Havlova, M., Gubis, J., Vankova, R.: Comparison of hormonal responses to heat, drought and combined stress in tobacco plants with elevated proline content. - J Plant Physiol. 167: 1360-1370, 2010.

Egamberdieva, D., Li, L., Lindström, K., Räsänen, L.A.: A synergistic interaction between salt-tolerant Pseudomonas and Mesorhizobium strains improves growth and symbiotic performance of liquorice (Glycyrrhiza uralensis Fisch.) under salt stress. - Appl. Microbiol. Biotechnol. 100: 2829-2841, 2016.

Epstein, E.: Silicon. - Annu. Rev. Plant Physiol. Plant mol. Biol. 50: 641-664, 1999.

Farooq, M.A., Ali, S., Hameed, A., Ishaque, W., Mahmood, K., Iqbal, Z.: Alleviation of cadmium toxicity by silicon is related to elevated photosynthesis, antioxidant enzymes; suppressed cadmium uptake and oxidative stress in cotton. - Ecol. Environ. Safety 96: 242-249, 2013.

Farooq, M., Wahid, A., Lee, D.J., Ito, O., Siddique, K.H.: Advance in drought resistance of rice. - Crit. Rev. Plant Sci. 28: $199-217,2009$.

Feng, J.P., Shi, Q.H., Wang, X.F., Wei, M., Yang, F.J., Xu, H.: Silicon supplementation ameliorated the inhibition of photosynthesis and nitrate metabolism by cadmium toxicity in Cucumis sativus L. - Sci. Hort. 123: 521-530, 2010.

Fernández-García, N., Olmos, E., Bardisi, E., Garma, G. D. L., López-Berenguer, C., Rubio-Asensio, J.S.: Intrinsic water use efficiency controls the adaptation to high salinity in a semiarid adapted plant, henna (Lawsonia inermis L.). - J. Plant Physiol. 171: 64-75, 2014.

Gao, X., Zou, C., Wang, L., Zhang, F.: Si decreases transpiration rate and conductance from stomata of maize plants. - J. Plant Nutr. 29: 1637-1647, 2006.

Gong, H., Chen, K.: The regulatory role of Si on water relations, photosynthetic gas exchange, and carboxylation activities of wheat leaves in field drought conditions. - Acta Physiol. Plant. 34: 1589-1594, 2012.

Haghighi, M., Pessarakli, M.: Influence of silicon and nanosilicon on salinity tolerance of cherry tomatoes (Solanum lycopersicum L.) at early growth stage. - Sci. Hort. 161: 111117, 2013.

Hajihashemi, S., Ehsanpour, A.A.: Influence of exogenously applied paclobutrazol on some physiological traits and growth of Stevia rebaudiana, under in vitro drought stress. - Biologia 68: 414-420, 2013.

Hashemi, A., Abdolzadeh, A., Sadeghipour, H. R.: Beneficial effects of silicon nutrition in alleviating salinity stress in hydroponically grown canola, Brassica napus L., plants. Soil Sci. Plant Nutr. 56: 244-253, 2010. 
Hattori, T., Inanaga, S., Araki, H., An, P., Mortia, S., Luxova, M., Lux, A.: Application of Si enhanced drought tolerance in Sorghum bicolor. - Physiol Plant. 123: 459-466, 2005.

Hattori, T., Sonobe, K., Inanaga, S., An, P., Morita, S.: Effects of silicon on photosynthesis of young cucumber seedlings under osmotic stress. - J. Plant Nutr. 31: 1046-1058, 2008.

He, Q., Dong, S., Gao, R.: Relationship between development of spike vascular bundle and sink capacity of ear and kernel in maize (Zea Mays L.). - Acta agron. sin. 31: 995-1000, 2005.

Huang, Z., Zou, Z., He, C., He, Z., Zhang, Z., Li, J.: Physiological and photosynthetic responses of melon (Cucumis melo L.) seedlings to three glomus species under water deficit. - Plant Soi. 339: 391-399, 2011.

Janz, D., Lautner, S., Wildhagen, H., Behnke, K., Schnitzler, J.P., Rennenberg, H., Fromm, J., Polle, A.: Salt stress induces the formation of a novel type "pressure wood" in two Populus species. - New Phytol. 194: 129-141, 2012.

Jaleel, C.A., Manivannan, P., Wahid, A., Farooq, M., Al-Juburi, H.J., Somasundaram, R., Panneerselvam, R.: Drought stress in plants: a review on morphological characteristics and pigments composition. - Int. J. Agr. Biol. 11: 100-105, 2009.

Katz, O.: Beyond grasses: the potential benefits of studying silicon accumulation in non-grass species. - Front Plant Sci. 5: 367, 2014.

Kavas, M., Akça, O.E., Akçay, U.C., Peksel, B., Eroğlu, S., Öktem, H.A., Yücel, M.: Antioxidant responses of peanut (Arachis hypogaea L.) seedlings to prolonged salt-induced stress. - Arch. biol. Sci. 671: 303-312, 2015.

Keremu, Y., Hou, J.T.: [Effects of salt stress on microstructure and ultrastructure of glycophytes.] - Xinjiang agr. Sci. 43: 234-236, 2006. [In Chin.]

Kraus, T.E., Mckersie, B.D., Fletcher, R.A.: Paclobutrazole induced tolerance of wheat leaves to paraquat may involve antioxidant enzyme activity. - J. Plant Physiol. 145: 570-576, 1995.

Li, M., Wang, G.: Effect of drought stress on activities of cell defense enzymes and lipid peroxidation in Glycyrrhiza uralensis seedings. - Acta ecol. Sin. 22: 503-507, 2002.

Li, Q., Ma, C., Li, H., Xiao, Y., Liu, X.: Effects of soil available silicon on growth, development and physiological functions of soybean. - Chin. J. appl. Ecol. 15: 73-76, 2004.

Li, R., Shi, F., Fukuda, K., Yang, Y.: Effects of salt and alkali stresses on germination, growth, photosynthesis and ion accumulation in alfalfa (Medicago sativa L.). - Soil Sci. Plant Nutr. 56: 725-733, 2010.

Li, Y.T., Zhang, W.J., Cui, J.J., Lang, D.Y., Li, M., Zhao, Q.P., Zhang, X.H.: Silicon nutrition alleviates the lipid peroxidation and ion imbalance of Glycyrrhiza uralensis seedlings under salt stress. - Acta Physiol. Plant. 38: 96-105, 2016.

Liang, Y., Zhu, J., Li, Z., Chu, G., Ding, Y., Zhang, J., Sun, W.: Role of $\mathrm{Si}$ in enhancing resistance to freezing stress in two contrasting winter wheat cultivars. - Environ. exp. Bot. 64: 286-294, 2008.

Liang, Y.C., Zhang, W.Q., Chen, J., Ding, R.: Effect of Si on $\mathrm{H}^{+}$-ATPase and $\mathrm{H}^{+}$-PPase activity, fatty acid composition and fluidity of tonoplast vesicles from roots of salt stressed barley (Hordeum vulgare L.). - Environ. exp. Bot. 53: 29-37, 2005.

Liu, Y., Yue, X., Chen, G.L.: Effects of water stress on ultrastructure and membrane lipid peroxidation of leaf and root cells of Glycyrrhiza uralensis. - Acta Prataculturae sin. 19: 79-86, 2010.

Lobato, A.K.S., Coimbra, G.K., Neto, M.A.M., Costa, R.C.L., Santos Filho, B.G., Oliveira Neto, C.F., Luz, L.M., Barreto, A.G.T., Pereira, B.W.F., Alves, G.A.R., Monteiro, B.S., Marochio, C.A.: Protective action of silicon on relations and photosynthetic pigments in pepper plants induced to water deficit. - Res. J. biol. Sci. 4: 617-623, 2009.

Ma, D., Sun, D., Wang, C., Qin, H., Ding, H., Li, Y., Guo, T.: Silicon application alleviates drought stress in wheat through transcriptional regulation of multiple antioxidant defense pathways. - J. Plant Growth Regul. 35: 1-10, 2016.

Ma, J.F., Yamaji, N.: Function and transport of silicon in plants. - Cell Mol. Life Sci. 65: 3049-3057, 2008.

Ma, Q., Yue, L.J., Zhang, J.L., Wu, G.Q., Bao, A.K., Wang, S.M.: Sodium chloride improves photosynthesis and water status in the succulent xerophyte Zygophyllum xanthoxylum. - Tree Physiol. 32: 4, 2012.

Maghsoudi, K., Emam, Y., Pessarakli, M.: Effect of silicon on photosynthetic gas exchange, photosynthetic pigments, cell membrane stability and relative water content of different wheat cultivars under drought stress conditions. - J. Plant Nutr. 39: 1001-1015, 2016.

Maghsoudi, K., Maghsoudi, A.: Analysis of the effects of stomatal frequency and size on transpiration and yield of wheat (Triticum aestivum L.). - Amer. Eur. J. agr. environ. Sci. 3: 865-872, 2008 .

Mazumdar, J.: Phytoliths of pteridophytes. - S. Afr. J. Bot. 77: 10-19. 2011

Ming, H., Hu, C.S., Zhang, Y.M., Cheng, Y.S.: Improved extraction methods of chlorophyll from maize. - J. Maize Sci. 15: 93-95, 2007.

Moussa, H.R.: Influence of exogenous application of $\mathrm{Si}$ on physiological response of salt-stressed maize (Zea mays L.). Int. J. Agr. Biol. 2: 293-297, 2006.

Muneer, S., Jeong, B.R.: Proteomic analysis of salt-stress responsive proteins in roots of tomato (Lycopersicon esculentum L.) plants towards silicon efficiency. - Plant Growth Regul. 77: 133-146, 2015.

Noode'n, L.D., Guiame't, J.J., John, I.: Whole plant senescence. - In: Noode'n, L.D., (ed.): Plant Cell Death Processes. Pp. 227-244. Academic Press, San Diego 2004.

Olmos, E., Sánchez-Blanco, M.J., Ferrández, T., Alarcón, J.J.: Subcellular effects of drought stress in Rosmarinus officinalis. - Plant Biol. 9: 7-84, 2007.

Oksanen, E., Riikonen, J., Kaakinen, S., Holopainen, T., Vapaavuori, E.: Structural characteristics and chemical composition of birch (Betula pendula) leaves are modified by increasing $\mathrm{CO}_{2}$ and ozone. - Global Change Biol. 11: 732-748, 2005.

Ouzounidou, G., Ilias, I.F., Giannakoula, A., Theocharidou, I.: Effect of water stress and $\mathrm{NaCl}$ triggered changes on yield, physiology, biochemistry of broad bean (Vicia faba L.) plants and on quality of harvested pods. - Biologia 69: 1010-1017. 2014.

Peng, Y.Y., Peng, Z.S., Song, H.X., Xu, J.S.: Chromosomal location of the genes associated with photosynthesis of Lophopyrum elongatum, (host) a. löve in chinese spring background. - J. int. Agr. 5: 579-586, 2006.

Pilon-Smits, E.A., Quinn, C.F., Tapken, W., Malagoli, M., Schiavon, M.: Physiological functions of beneficial elements. - Curr. Opin. Plant Biol. 12: 267-274, 2009.

Ping, M.A., Bai, T.H., Wang, X.Q.: Effects of light intensity on photosynthesis and photoprotective mechanisms in apple under progressive drought. - J. int. Agr. 14: 1755-1766, 2015.

Porcel, R., Redondo-Gómez, S., Mateos-Naranjo, E., Aroca, R., Garcia, R., Ruiz-Lozano, J.M.: Arbuscular mycorrhizal symbiosis ameliorates the optimum quantum yield of photosystem II and reduces non-photochemical quenching in rice plants subjected to salt stress. - J. Plant Physiol. 185: 7583, 2015.

Rizwan, M., Ali, S., Ibrahim, M., Farid, M., Adrees, M., Bharwana, S.A., Zia-ur-Rehman, M., Qayyum, M.F., Abbas, 
F.: Mechanisms of silicon-mediated alleviation of drought and salt stress in plants: a review. - Environ. Sci. Pollut. Rev. 22: 1-16, 2015.

Shahid, M.A., Balal, R.M., Pervez, M.A., Abbas, T., Aqeel, M.A., Javaid, M.M., Garcia-Sanchez, F.: Exogenous proline and proline-enriched Lolium perenne leaf extract protects against phytotoxic effects of nickel and salinity in Pisum sativum by altering polyamine metabolism in leaves. - Turk. J. Bot. 38: 914-926, 2014.

Shen, X., Zhou, Y., Duan, L., Li, Z., Eneji, A.E., Li, J.: Si effects on photosynthesis and antioxidant parameters of soybean seedlings under drought and ultraviolet-B radiation. - J. Plant Physiol. 167: 1248-1252, 2010.

Silva, O.N., Lobato, A.K.S., Avila, F.W., Costa, R.C.L., Oliveira Neto, C.F., Santos Filho, B.G., Martins Filho, A.P., Lemos, R.P., Pinho, J.M., Medeiros, M.B.C.L., Cardoso, M.S., Andrade, I.P.: Silicon-induced increase in chlorophyll is modulated by the leaf water potential in two water-deficient tomato cultivars. - Plant Soil Environ. 58: 481-486, 2012.

Sonobe, K., Hattori, T., An, P., Tsuji, W., Eneji, A.E., Kobayashi, S., Kawamura, Y., Tanaka, K., Inanaga, S.: Effect of silicon application on sorghum root responses to water stress. - J. Plant Nutr. 34: 71-82, 2011.

Sultana, N., Ikeda, T., Itoh, R.: Effect of $\mathrm{NaCl}$ salinity on photosynthesis and dry matter accumulation in developing rice grains. - Environ. exp. Bot. 42: 211-220, 1999.

Teulat, B., Zoumarou-Wallis, N., Rotter, B., Ben Salem, M., Bahri, H., This, D.: QTL for relative water content in fieldgrown barley and their stability across Mediterranean environments. - Theor. appl. Genet. 108: 181-188, 2003.

Wang, S., Liu, P., Chen, D., Yin, L., Li, H., Deng, X.: Silicon enhanced salt tolerance by improving the root water uptake and decreasing the ion toxicity in cucumber. - Front Plant Sci. 6: 759, 2015.

Xu, P.L., Guo, Y.K., Bai, J.G., Shang, L., Wang, X.J.: Effects of long-term chilling on ultrastructure and antioxidant activity in leaves of two cucumber cultivars under low light. - Physiol. Plant. 132: 467-478, 2008.

Xu, Z.Z., Zhou, G.S., Shimizu, H.: Effects of soil drought with nocturnal warming on leaf stomatal traits and mesophyll cell ultrastructure of a perennial grass. - Crop Sci. 49: 1843-1851, 2009.

Yang, X.H., Li, J.M., Dong, X.H., Duan, L.S., Li, Z.H.: Effects of salt stress on growth and some physiological indexes in fisch seedlings. - Acta agr. boreali-sin. 21: 39-42, 2006.

Yin, L., Wang, S., Li, J., Tanaka, K., Oka, M.: Application of silicon improves salt tolerance through ameliorating osmotic and ionic stresses in the seedling of Sorghum bicolor. - Acta Physiol. Plant. 35: 3099-3107, 2013.

Zellnig, G., Zechmann, B., Perktold, A.: Morphological and quantitative data of plastids and mitochondria within droughtstressed spinach leaves. - Protoplasma 223: 221-227, 2004.

Zhang, L., Zhang, L., Sun, J., Zhang, Z., Ren, H., Sui, X. Rubisco gene expression and photosynthetic characteristics of cucumber seedlings in response to water deficit. - Sci. Hort. 161: 81-87, 2013.

Zhang, W.J., Xie, Z.C., Wang, L.H., Li, M., Lang, D.Y., Zhang, X.H.: Silicon alleviates salt and drought stress of Glycyrrhiza uralensis seedling by altering antioxidant metabolism and osmotic adjustment. - J. Plant Res. 130: 611-624, 2017.

Zhu, Y., Gong, H.: Beneficial effects of silicon on salt and drought tolerance in plants. - Agron. sustain. Dev. 34: 455-472, 2014. 